\title{
Beyond 3 neutrino oscillations
}

\author{
ANDRÉ DE GOUVÊA ${ }^{* \dagger}$ \\ Northwestern University \\ E-mail: degouvea@northwestern.edu
}

\begin{abstract}
All solar, atmospheric, reactor and long-baseline accelerator neutrino data can be explained by postulating that there are three neutrinos, that these have non-zero, non-identical masses, and that the neutrino mass eigenstates are not aligned with the neutrino flavor eigenstates. In this talk, I first briefly discuss experimental data - along with possible interpretations - that may already point to physics beyond the minimal massive neutrino paradigm. I then discuss the possibility that there are more neutrinos than the old standard model called for. These so-called sterile neutrinos are a very simple and often quite useful extension of the standard model, and may prove to be a potentially testable side-effect of the physics responsible for neutrino masses.
\end{abstract}

10th International Workshop on Neutrino Factories, Super beams and Beta beams

June 30 - July 52008

Valencia, Spain

\footnotetext{
* Speaker.

$\dagger$ It is a pleasure to thank the Local Organizing Committee of NuFact'08 for putting together an excellent program in a very relaxing and inspiring venue. I also thank Carlos Peña-Garay and the Scientific Program Committee for the invitation. This work is sponsored in part by the US Department of Energy Contract DE-FG02-91ER40684.
} 


\section{Introduction}

After several decades of confusion, neutrino oscillation data over the past ten years have revealed, beyond reasonable doubt, that neutrinos have mass and that leptons mix $[1,2,3,4,5]$. Almost all data from atmospheric, rector and accelerator neutrino experiments are consistent with the hypothesis that there are three neutrinos and that the standard model electroweak interactions properly describe all neutrino-associated processes.

Current and next-generation neutrino experiments $[3,4,5,6]$ aim at definitively testing the three massive active neutrino hypothesis and, in the process, determining the "left-over" neutrino oscillation parameters ( $\theta_{13}$, the CP-odd phase $\delta$ and the neutrino mass hierarchy). While almost all detailed discussions surrounding next-generation neutrino experiments concentrate on how well neutrino oscillation parameters can be measured or constrained, there is the possibility that "more" new physics, beyond massive neutrinos, can manifest itself in neutrino oscillations. The two most palatable possibilities are new, "weaker-than-weak" neutrino-matter interactions, discussed in [7] and new light neutral fermionic degress of freedom - sterile neutrinos. The latter are the subject of this talk.

Sterile neutrinos are often associated to the so-called LSND anomaly [8]. Over the past couple of years, new light was shed over this puzzle by the MiniBooNE experiment (see [9] and references therein). A very brief summary of what we learned from MiniBooNE results is presented in Sec. 2. Sterile neutrinos are properly introduced and discused, independently from the LSND anomaly, in Sec. 3. Section 4 offers some concluding remarks.

\section{LSND and MiniBooNE: a Theorist's Abridged Overview}

The LSND experiment looked for an excess of $\bar{v}_{e}$ candidates from a neutrino "beam" originated from positively charged pion decay in flight $\left(\pi^{+} \rightarrow \mu^{+} v_{\mu}\right)$ and anti-muon decay at rest $\left(\mu^{+} \rightarrow e^{+} v_{e} \bar{v}_{\mu}\right)$. Typical LSND neutrino energies are around $50 \mathrm{MeV}$. The experiment observes a statistically significant excess of $\bar{v}_{e}$ events. The excess is consistent with a tiny probability $\left(P_{\mu e}=(0.26 \pm 0.08) \%\right)$ that $\bar{v}_{\mu} \rightarrow \bar{v}_{e}$ after propagating around 30 meters [8]. The physics responsible for this excess, however, has yet to be unambiguously observed by an independent experimental effort.

The MiniBooNE experiment studied neutrinos produced in positively charged pion decay in flight $\left(\pi^{+} \rightarrow \mu^{+} v_{\mu}\right)$ and looked for an excess of $v_{e}$ candidates. MiniBooNE energies range from, roughly, $200 \mathrm{MeV}$ to $2 \mathrm{GeV}$ and the source-detector distance was chosen such that the typical values of $L / E$ were the same at MiniBooNE and LSND ([9] and references therein). MiniBooNE results, combined with the world's neutrino data, are not consistent with a neutrino oscillation interpretation to the LSND anomaly, as I try to summarize below. The MiniBooNE data is, however, not consistent with standard model expectations for low neutrino energies ( $E \lesssim 450 \mathrm{MeV}$ ) [10]. I'll comment on this low-energy excess of events at MiniBooNE shortly.

One often refers to a toy model in order to discuss the compatibility of LSND with (some of the) other neutrino oscillation experiments. This so-called two-flavor interpretation of the LSND anomaly assumes that $P_{\mu e}=\sin ^{2} 2 \theta_{\mathrm{LSND}} \sin ^{2}\left(\Delta m_{\mathrm{LSND}}^{2} L / 4 E\right)$. At the $90 \%$ confidence level, there is no value of $\theta_{\mathrm{LSND}}, \Delta m_{\mathrm{LSND}}^{2}$ consistent with both MiniBooNE and LSND data. 
In the real world, however, the result above is only of limited interest. After all, one needs to account for all positive and negative searches for neutrino oscillations, and there are at least three neutrinos. One can quickly check that, with three neutrinos, it is impossible to explain all neutrino data with those from LSND even if one ignores the results from MiniBooNE. The reason is quite simple: three very different oscillation frequencies - associated to three different mass-squared differences: $\Delta m_{\text {solar }}^{2} \sim 10^{-4} \mathrm{eV}^{2}, \Delta m_{\mathrm{atm}}^{2} \sim 10^{-3} \mathrm{eV}^{2}$ and $\Delta m_{\mathrm{LSND}}^{2} \gtrsim 10^{-1} \mathrm{eV}^{2}-$ are required to explain all data. Three massive neutrinos, however, only allow for two independent mass-squared differences.

With four neutrinos the situation is more complicated but it fair to say that there is no good fit to all data. Given the three required mass-squared differences, the neutrino masses can be ordered in two qualitatively different (as far as oscillations are concerned) ways, the so-called $2+2$ neutrino mass ordering, and the so-called $3+1$ neutrino mass ordering (see, for example, [11]). The $2+2$ mass ordering is severely disfavored by solar and atmospheric neutrino oscillation data [11] , and will be ignored henceforth. The $3+1$ neutrino mass ordering provides a mediocre fit to all data before MiniBooNE, and is ruled out, according to [12], at more than the $99 \%$ confidence level once MiniBooNE data are taken into account. ${ }^{1}$

With five neutrinos, a $3+1+1$ mass ordering provides the best fit to all data (for an early analysis and more details, see [13]), including those from MiniBooNE. According to [12], however, the goodness of the fit is very poor ("ruled out" at the three sigma level [12]). It is interesting that if only positive oscillation results (including LSND and the low-energy excess at MiniBooNE) are considered, a healthy $3+1+1$ solution can be constructed. More interesting is the fact that the best-fit solution relies non-trivially on CP-violating effects, which render LSND $\bar{v}_{\mu}$ oscillations more pronounced than MiniBooNE $v_{\mu}$ oscillations [12]. Unfortunately, such a solution is severely constrained by negative searches for neutrino oscillations. It is fair to say, however, that a positive result from the MiniBooNE antineutrino data, consistent with LSND, would have rendered this solution worthy of careful scrutiny. According to preliminary MiniBooNE antineutrino results still statistically limited - this appears not to be the case [14].

In summary, it is fair to say that there is no neutrino oscillation solution to all neutrino data (including those from LSND and MiniBooNE) regardless of how many light neutrinos exist. This does, not, however, mean that there is no new physics interpretation of all neutrino data. Instead of reviewing all the different solutions in the market, I'll qualitatively describe what requirements these solutions should meet (for a more detailed discussion along these lines, see [15]). I start by reminding readers that the LSND effect is very small (remember, it requires $P_{\mu e} \sim 0.3 \%$ ) and that few experiments have the same sensitivity to the same observable. The fact that the Karmen II experiment [16] does not observe an LSND-like effect, however, rules out the possibility that the LSND signal is baseline $(L)$ independent as the two experiments have similar sensitivity and identical beams and the main distinguishing feature is that $L_{\mathrm{LSND}}>L_{\mathrm{Karmen}}$. On the other hand, the fact that there is no excess at high energies in MiniBooNE rules out the possibility that the solution to LSND is proportional to $L / E .^{2}$ We are hence forced into new physics effects that depend on the baseline $L$ and neutrino energy $E$ but does not depend only on the ratio $L / E$. This is

\footnotetext{
${ }^{1}$ This is one circumstance where the two-flavor oscillation approximation applies for comparing MiniBooNE and LSND.

${ }^{2}$ Strictly speaking, CP-violating effects can by-pass this constraint. This possibility will be ignored for simplicity.
} 
challenging for two reasons. One is that Lorentz invariance states that the $L$ dependency scales like $L / E$ (think Lorentz contraction of $L$ ). Hence we are pushed towards Lorentz-invariant violating effects (either "medium-induced" or intrinsic). Second, the dependency on $L$ and $E$ must be such that in other neutrino oscillation experiments the small LSND effect is not significantly larger than a few percent. Since we have performed experiments with a variety of neutrino energies (from $1 \mathrm{MeV}$ to $10+\mathrm{GeV}$ ) and baselines (from 10 meters to 1 astronomical unit), solutions to the LSND result have to be Lorentz violating, restricted to neutrino energies around $100 \mathrm{MeV}$, and "optimal" for baselines around 100 meters. One "existence proof" solution can be found in [15] and a few other logical possibilities have been proposed. It is fair to say that no solution has been deemed acceptable by any reasonably sized subset of the particle physics community.

Before moving on, I'd like to comment on the low-energy excess observed in the MiniBooNE neutrino data. It could be a manifestation of a poorly understood or neglected standard model process. In [17], for example, it was proposed that the cross-section for $v+N \rightarrow v+N+\gamma$ might be significantly enhanced at MiniBooNE low-energies by a baryon-number anomaly Chern-Simonslike term that connects a baryon-number current (say, $N \rightarrow N$ ) to a photon and a Z-boson. Details are still under construction, but this hypothesis could be verified with a detector capable of distinguishing electrons from photons (such as microBooNE [18]).

\section{Sterile Neutrinos}

Irrespective of the LSND anomaly, sterile neutrino are among the simplest extensions to the standard model of particle physics. Now that it has been determined that neutrinos have nonzero masses, sterile neutrinos are also among the most likely ones to be realized in Nature. Before proceeding, let me define 'sterile neutrinos.' Sterile neutrinos are fermions that transform trivially under the standard model gauge symmetries, i.e., they are gauge singlet fermions. If no other new degrees of freedom exist, sterile neutrinos $N_{i}(i=1,2, \ldots)$ can only couple to the standard model fields (at the renormalizable level) via a neutrino Yukawa coupling interaction: $\mathscr{L}_{\text {int }}=\lambda_{\alpha i} L_{\alpha} N_{i} H$, where $L_{\alpha}(\alpha=e, \mu, \tau)$ are the lepton-doublet fields and $H$ is the Higgs doublet field.

Here I concentrate on light sterile neutrinos (masses well below the weak scale). If $\lambda$ are small and the sterile neutrinos light, sterile neutrinos manifest themselves via mixing. This is easy to see. After electroweak symmetry breaking, the sterile neutrinos mix with the active neutrinos via a mixing angle proportional to $\lambda v$, where $v=246 \mathrm{GeV}$ is the Higgs boson vacuum expectation value.

While one can argue for the existence sterile neutrinos, there is no water-tight argument for the existence of light sterile neutrinos. Typical arguments against light sterile neutrinos include "what are sterile neutrinos good for?," "why would a gauge-singlet fermion be very light?," and "what does a typical sterile neutrino look-like, i.e. what should we expect sterile neutrino masses and couplings to be?." I'll address some of these complaints in what follows, and will discuss one concrete, well-known example.

Instead of asking what sterile neutrinos are good for, it is more productive to ask a related question: if there are gauge singlet degrees of freedom (these are the members of a so-called hidden sector), how do we get to "see" them? The answer is that, unless there are also new standardmodel-charged degrees of freedom, hidden sector particle can couple to the standard model in only 
two ways. Singlet scalar fields $S$ can couple to the standard model Higgs field (via, say, $S|H|^{2}$ and $S^{2}|H|^{2}$ interactions). Singlet fermion fields - sterile neutrinos - couple to the lepton doublet and the Higgs doublet via the Yukawa interaction described above. Hence, neutrinos and the Higgs boson provide the only windows to hidden sector particles (assuming they exist). Furthermore, hidden sector particle - sterile neutrinos in particular - may play a big role when it comes to addressing some of the big questions being addressed by fundamental physics today. For example, gauge singlet particles, including sterile neutrinos, are the among the best candidates for dark matter.

As alluded to above, sterile neutrinos are also a natural "side effect" of active neutrino masses. Consider the standard model augmented by three sterile neutrino fields. The most general renormalizable Lagrangian $\mathscr{L}_{v}$ consistent with gauge invariance is

$$
\mathscr{L}_{v}=\mathscr{L}_{\text {old }}-\lambda_{\alpha i} L_{\alpha} H N_{i}-\sum_{i=1}^{3} \frac{M_{i}}{2} N_{i} N_{i}+H . c .,
$$

where $M_{i}$ are three Majorana neutrino masses for the $N_{i}$ fields, which are taken to be Weyl fermions. After electroweak symmetry breaking, Eq. (3.1) describes six neutral fermions and can easily describe all neutrino data (excluding LSND and MiniBooNE) for a very wide range of "new physics parameters" $\lambda$ and $M$.

A summary of the situation is as follows. If all $M_{i}=0$, the six neutrinos "fuse" into three Dirac fermions with a mass matrix $\mu=\lambda v$. In this case, $\mathscr{L}_{v}$ contains a $U(1)_{B-L}$ global symmetry (which is explicitly broken in the case $M \neq 0$ ). This indicates that any value of $M$ is technically natural, i.e., quantum corrections to $M$ are proportional to $M$ itself and only logarithmically sensitive to any ultraviolet energy scale.

If, on the other hand, $M \gg \mu$, the seesaw mechanism [19] is realized. ${ }^{3}$ In this case, the six neutrino mass eigenstates split into two triplets, one mostly made up of the active neutrinos $\left(v_{e, \mu, \tau}\right)$, the other mostly made up of the sterile neutrinos $\left(v_{s_{1}, s_{2}, s_{3}}\right)$. The Majorana mass matrix of the mostly active states is $m_{\alpha \beta}=\sum_{i} \mu_{\alpha_{i}} M_{i}^{-1} \mu_{\beta i}$, while the mostly sterile states have masses $M_{i}$ (plus $\mu M^{-1}$ corrections). Data have revealed that the mostly active neutrino masses are $m_{v} \lesssim 10^{-1} \mathrm{eV}$. The seesaw mechanism is, therefore, operational as long as $M>$ few $\mathrm{eV}$ (this possibility was first emphasized in [20]). Effects of the mostly sterile states depend dramatically on the value of $M$ and $\lambda$. Some consequences are explored in [20, 21, 22] (and see many references therein) for $M_{i} \lesssim 10 \mathrm{MeV}$. The most important fact is that, naively, there is a relationship between the neutrino masses and the active-sterile mixing angles. Hence, we can address two of the criticisms raised above. One is that sterile neutrinos have a "purpose." They are an integral component of the physics responsible for neutrino masses. The other is that we expect the active-sterile mixing angles

$$
\left|U_{\alpha(i+3)}\right|^{2} \sim \frac{m_{v}}{M_{i}}
$$

where $m_{v}$ is a linear combination of the active neutrino masses and we assume all $M_{i}$ are much larger than the active neutrino masses. Hence, there is the possibility to either rule out or strengthen the case for Eq. (3.1) experimentally as long as the mixing angles in Eq. (3.2) are not too tiny, i.e. $M_{i}$ are not too large. For more details see, for example, [21,23]. Expectations for $U_{\alpha(i+3)}$ are depicted

\footnotetext{
${ }^{3}$ There are two other possibilities, which I won't discuss. The case $M \sim \mu$ is disfavored by neutrino oscillation experiments. The case $M \ll \mu$ leads to quasi-Dirac neutrinos.
} 


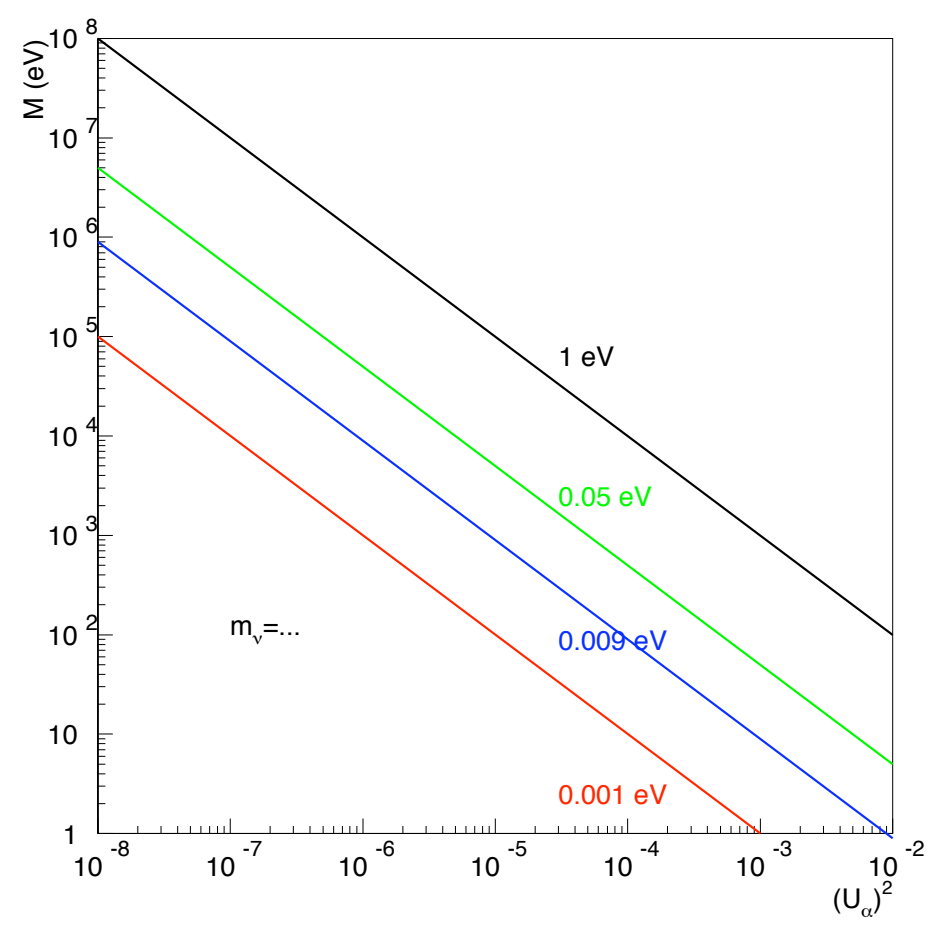

Figure 1: Expectations for active sterile-mixing as a function of the right-handed neutrino masses, for different values of the effective active neutrino mass.

in Fig. 1. For light sterile neutrinos (small $M$ ), active-sterile neutrino mixing can be sizeable and potentially observable in a variety of neutrino experiments. If $M \sim 1-10 \mathrm{eV}$, one expects to "see" sterile neutrinos in oscillation experiments. For $M \sim 1-10 \mathrm{keV}$ (and $m_{v} \ll 0.001 \mathrm{eV}$ ), sterile neutrinos qualify as an interesting warm dark matter candidate [22]. For $M \gtrsim 100 \mathrm{MeV}$, sterile neutrino effects are negligible at laboratory experiments unless $m_{v}$ in Eq. (3.2) turns out to be much larger than the active neutrino masses. This possibility was recently explored in [24, 23].

Before concluding, let me discuss the origin of small active neutrino masses in the case of the seesaw, emphasizing the distinction between a high-energy seesaw mechanism $(M \gg 100 \mathrm{GeV})$ from a low-energy one $(M \ll 1 \mathrm{GeV})$. In both cases, the active neutrino masses are proportional to $\left(\lambda^{2} / M\right) \times v^{2}$. In the high-energy seesaw, neutrino masses are small because the new physics responsible for neutrino masses is very heavy: $m_{v} \propto M_{\text {weak }} / M_{\text {new }}$. In the low-energy seesaw, neutrino masses are small because the physics responsible for neutrino masses is very weakly coupled: $m_{v} \propto \lambda_{\text {new }}$. The only way to distinguish the two qualitatively different possibilities is experimentally and, fortunately, the low-energy option seems to be falsifiable!

\section{Concluding Remarks}

I have reviewed two loosely connected topics: the status of the LSND anomaly and the possibility that there are light sterile neutrinos.

As far as the LSND anomaly is concerned, MiniBooNE results have ruled out most "standard" solutions to the LSND anomaly, including those involving light sterile neutrinos. On the other 
hand, MiniBooNE results contain a puzzle that still needs to be resolved - what is the physics responsible for the low-energy excess of $v_{e}$ candidates?

"Non-standard" solutions to the LSND anomaly, including the recent MiniBooNE results, are alive and well. All solutions, however, are taylor-made and require multiple "new physics" effects (say, sterile neutrinos and CPT violation). There is no "feel good" solution to the LSND puzzle. This is especially true since the first MiniBooNE results became available.

On the other hand, gauge singlet fermions (sterile neutrinos) are a simple, benign extension to the standard model. regardless of their mass, these manifest themselves only through their couplings to the lepton doublets and the Higgs boson doublet. In the case of light gauge singlet fermions, the only way we have of detecting their existence is through their mixing with the weaklyinteracting neutrinos. While there is no guarantee that light sterile neutrinos exist, they may play a significant role in the universe. Light sterile neutrinos are a good dark matter candidate and may provide us indispensable clues regarding the origin of neutrino masses.

I conclude by reminding readers of the primary goal of next-generation neutrino oscillation experiments (the main purpose of this conference series!): to scrutinize the current neutrino oscillation paradigm, i.e., the hypothesis that there are three massive neutrinos which couple to the $W$ and $Z$ bosons as dictated by the $S U(2) \times U(1)$ electroweak interactions. It is imperative to ask what new physics can lie behind the current neutrino oscillation paradigm. I believe that light sterile neutrinos are among the most realistic and exciting (and very informative) possibilities.

\section{References}

[1] H. Murayama, Neutrinos in the Standard Model and Beyond, , in proceedings of 10th International Workshop on Neutrino Factories, Super beams and Beta beams (NuFact08), June 30 - July 5 2008, Valencia, Spain POS (NUFACT08) 001.

[2] M. C. Gonzalez-Garcia, Physics of Massive Neutrinos, in proceedings of 10th International Workshop on Neutrino Factories, Super beams and Beta beams (NuFact08), June 30 - July 5 2008, Valencia, Spain POS (NUFACT08) 002.

[3] N. Saoulidou, Status and Prospects for Long Baseline Experiments, in proceedings of 10th International Workshop on Neutrino Factories, Super beams and Beta beams (NuFact08), June 30 July 5 2008, Valencia, Spain PoS (NUFACT08) 003.

[4] T. Kajita, Status and Prospects for Solar and Atmospheric Neutrinos, in proceedings of 10th International Workshop on Neutrino Factories, Super beams and Beta beams (NuFact08), June 30 July 5 2008, Valencia, Spain PoS (NUFACT08) 006.

[5] D. Lhuillier, Status and Prospects for Reactor Experiments, in proceedings of 10th International Workshop on Neutrino Factories, Super beams and Beta beams (NuFact08), June 30 - July 5 2008, Valencia, Spain POS (NUFACT08) 024.

[6] D. Harris and A. Jansson, Project X and Its Connection to Neutrino Physics, in proceedings of 10th International Workshop on Neutrino Factories, Super beams and Beta beams (NuFact08), June 30 July 5 2008, Valencia, Spain PoS (NUFACT08) 012.

[7] O. Yasuda, Non Oscillation Flavor Physics at Future Neutrino Oscillation Facilities, in proceedings of 10th International Workshop on Neutrino Factories, Super beams and Beta beams (NuFact08), June 30 - July 5 2008, Valencia, Spain PoS (NUFACT08) 016. 
[8] see A. Aguilar et al. [LSND Collaboration], Phys. Rev. D 64, 112007 (2001)and references therein.

[9] A. A. Aguilar-Arevalo et al. [The MiniBooNE Collaboration], Phys. Rev. Lett. 98, 231801 (2007); M. H. Shaevitz for the MiniBooNE Collaboration, J. Phys. Conf. Ser. 120, 052003 (2008).

[10] A. A. Aguilar-Arevalo et al. [The MiniBooNE Collaboration], arXiv:0812.2243 [hep-ex].

[11] M. Maltoni, T. Schwetz, M. A. Tortola and J. W. F. Valle, Nucl. Phys. B 643, 321 (2002) and references therein.

[12] M. Maltoni and T. Schwetz, Phys. Rev. D 76, 093005 (2007).

[13] M. Sorel, J. M. Conrad and M. Shaevitz, Phys. Rev. D 70, 073004 (2004).

[14] G. Karagiorgi for the MiniBooNE Collaboration, Joint Experimental-Theoretical Seminar, Fermilab, December 11, 2008. See http://theory. fnal.gov/jetp/.

[15] A. de Gouvêa and Y. Grossman, Phys. Rev. D 74, 093008 (2006).

[16] B. Armbruster et al. [KARMEN Collaboration], Phys. Rev. D 65, 112001 (2002).

[17] J. A. Harvey, C. T. Hill and R. J. Hill, Phys. Rev. Lett. 99, 261601 (2007).

[18] http://www-microboone.fnal.gov/.

[19] P. Minkowiski, Phys. Lett. B 67, 421 (1977); M. Gell-Mann, P. Ramond and R. Slansky in Supergravity, eds. D. Freedman and P. Van Niuenhuizen (North Holland, Amsterdam, 1979), p. 315; T. Yanagida in Proceedings of the Workshop on Unified Theory and Baryon Number in the Universe, eds. O. Sawada and A. Sugamoto (KEK, Tsukuba, Japan, 1979); S.L. Glashow, 1979 Cargèse Lectures in Physics - Quarks and Leptons, eds. M. Lévy et al. (Plenum, New York, 1980), p. 707; R.N. Mohapatra and G. Senjanović, Phys. Rev. Lett. 44, 912 (1980).

[20] A. de Gouvêa, Phys. Rev. D 72, 033005 (2005).

[21] A. de Gouvêa, J. Jenkins and N. Vasudevan, Phys. Rev. D 75, 013003 (2007).

[22] T. Asaka, S. Blanchet and M. Shaposhnikov, Phys. Lett. B 631, 151 (2005).

[23] A. de Gouvêa, arXiv:0706.1732 [hep-ph].

[24] J. Kersten and A. Yu. Smirnov, Phys. Rev. D 76, 073005 (2007). 